\title{
Control of tunneling by adapted signals
}

\author{
B. I. Ivlev \\ Department of Physics and Astronomy \\ University of South Carolina, Columbia, SC 29208 \\ and \\ Instituto de Física, Universidad Autónoma de San Luis Potosí \\ San Luis Potosí, S. L. P. 78000 Mexico
}

\begin{abstract}
Process of quantum tunneling of particles in various physical systems can be effectively controlled even by a weak and slow varying in time electromagnetic signal if to adapt specially its shape to a particular system. During an underbarrier motion of a particle such signal provides a "coherent" assistance of tunneling by the multi-quanta absorption resulting in a strong enhancement of the tunneling probability. The semiclassical approach based on trajectories in the complex time is developed for tunneling in a non-stationary field. Enhancement of tunneling occurs when a singularity of the signal coincides in position at the complex time plane with a singularity of the classical Newtonian trajectory of the particle. The developed theory is also applicable to the over-barrier reflection of particles and to reflection of classical waves (electromagnetic, hydrodynamic, etc.) from a spatially-smooth medium.
\end{abstract}

PACS numbers: 03.65.Sq, 42.50.Hz 


\section{INTRODUCTION}

Control of quantum systems by tuned external signals is an actively developed field at present, see for example [1] and references therein. Excitation of molecules, when one should excite only particular chemical bonds [2 [4, formation of programmable atomic wave packets [5], a control of electron states in heterostructures [6], and a control of photo-current in semiconductors [7] are typical examples of control by laser pulses. A control of quantum tunneling by electromagnetic signals is also a matter of interest, since tunneling is a part of many physical processes and of some chemical reactions. Modern facilities enable to tailor fast signals required for this purpose [8,9].

Let us focus on main aspects of tunneling under a non-stationary perturbation. The potential barrier $V(x)$, extended over the distance $a$, sets two typical energy scales: the barrier

hight $V_{0}$ and $\hbar \omega \sim \hbar \sqrt{V_{0} / m a^{2}}$, where $\omega$ can be associated with an oscillation frequency in the overturned potential. For semiclassical barriers the two energy scales are well separated $\hbar \omega \ll V_{0}$ and in absence of a non-stationary field the probability of tunneling through the barrier can be estimated with the exponential accuracy as $W \sim \exp \left(-V_{0} / \hbar \omega\right)$. In presence of the periodic signal $\mathcal{E}_{\Omega} \cos \Omega t$ a particle can absorb the quantum $\Omega$ with the probability $\left(a \mathcal{E}_{\Omega} / V_{0}\right)^{2}$ and tunnel in the more transparent part of the barrier with the probability $\exp \left(-\left(V_{0}-\Omega\right) / \hbar \omega\right)$. The total tunneling rate can be written as

$$
\begin{aligned}
W & \sim \exp \left(-\frac{V_{0}}{\hbar \omega}\right)+\left(\frac{a \mathcal{E}_{\Omega}}{V_{0}}\right)^{2} \exp \left(-\frac{V_{0}-\hbar \Omega}{\hbar \omega}\right) \\
& =\exp \left(-\frac{V_{0}}{\hbar \omega}\right)\left(1+\left(\frac{a \mathcal{E}_{\Omega}}{V_{0}}\right)^{2} \mathrm{e}^{\Omega / \omega}\right)
\end{aligned}
$$

Eq. 1 is approximate since in quantum mechanics one should multiply amplitudes but not probabilities, nevertheless the form (1) accounts necessary physical feature. Suppose a tunneling particle is acted by some electric field $\mathcal{E}(t)$ (periodic or pulse-type) and the typical time scale of this signal is $\theta$. Than one can distinguish two different physical situations: (i) hard signal, fast varying field with $\theta \sim \hbar / V_{0}$ and (ii) soft signal, slow varying field with $\theta \sim \omega^{-1}$. Tunneling can be governed easily by a hard signal even when its amplitude is 
less than the static barrier field $V_{0} / a$, since the probability of quantum absorption $\left(a \mathcal{E} / V_{0}\right)^{2}$ competes, according to Eq. 1, with the small tunneling rate (in this case $\Omega \sim \theta^{-1} \sim V_{0} / \hbar$ ). It is also obvious that a soft signal of the high amplitude $V_{0} / a$ (static field of the barrier) is able to govern tunneling. Can a soft signal with the amplitude much smaller than the static field of a potential barrier control effectively a tunneling process? Suppose a soft signal has the following shape

$$
\mathcal{E}(t)=\frac{\mathcal{E}}{\left(1+t^{2} / \theta^{2}\right)^{n}}
$$

Its Fourier harmonics $\mathcal{E}_{\Omega} \sim(\Omega \theta)^{n-1} \mathcal{E} \theta \exp (-\Omega \theta)$ should be inserted into Eq. 1. As follows from Eq. 1, when the signal width $\theta$ is less than $1 / 2 \omega$, the quadratic in $\mathcal{E}$ correction to the static probability diverges with increase of $\Omega$. It means the perturbation theory with respect to a weak non-stationary signal to break down for sufficiently short pulses. Note, the pulse still remains soft. This is an indication of efficiency of soft signals. Breaking of the perturbation theory means a significance of multi-quantum processes and a principal question is that what theory has to be used in this case.

A review of some aspects of tunneling in complex systems, including the instanton approach, was done in the book [10], see also Ref. [11]. Recent achievements in the semiclassical theory under stationary conditions are presented in Refs. 12 14. As it has been argued in Refs. [15, 16, 10], the semiclassical method of complex trajectories is applicable also to a non-stationary case, when a signal is periodic in time. Nevertheless, despite a number of publications, use of semiclassical theory for tunneling in a non-stationary field remains non-obvious. What happens in general case, in particular, for a short pulse like one given by the relation (2)? The goal of this work is to show that the semiclassical theory based on the concept of the complex time is an appropriate description of tunneling under action of a soft pulse of any shape. For the particular case of a triangular barrier the tunneling rate in presence of a non-stationary (soft) field is found to be determined, in the main exponential approximation, by the classical action $S(x, t)$ satisfying the Hamilton-Jacobi equation. In this approximation the wave function is proportional to $\exp (i S(x, t))$ (Below Planck's 
constant is unity). The first correction (preexponential) to this classical result and the second one were found explicitly and shown to be small comparing to the main contribution, what is typical for semiclassical approximation. The tunneling rate is found as a function of time, it tends to its static value at $t \rightarrow \pm \infty$, when $\mathcal{E}(t)=0$, and reaches the maximum at some moment of time. This maximum value is given, with an exponential accuracy, by an extreme value of the classical action, which is determined, according to classical mechanics, by means of classical trajectories. The classical trajectory obeys Newton's equation $m \partial^{2} x / \partial t^{2}+V^{\prime}(x)=\mathcal{E}(t)$ in the complex time, since in the real time there is no classical under-barrier path.

So, the method of classical trajectories in the complex time can be used, when the full time dependence of the tunneling rate is not required, but only its maximum value with an exponential accuracy is a matter of interest. Note, despite the classical action depends functionally on trajectories defined in the complex time plane, the argument $t$ of the action $S(x, t)$ is considered to be always real. The complex time has no physical meaning, it is only a possible way to parameterize a solution of the Hamilton-Jacobi equation. Nevertheless, this way is very useful. For a weak non-stationary field $\mathcal{E}(t)$ the classical trajectory $x_{0}(t)$ satisfies the unperturbed equation $(m / 2)\left(\partial x_{0} / \partial t\right)^{2}+V\left(x_{0}\right)=E$, where $E$ is the particle energy, which can identified with an incident energy of particle flux on the barrier $V(x)$. The classical trajectory $x_{0}(t)$, as a function of the complex $t$, has the singularity at $t=t_{s}(E)$ 15,16 and the external signal (2) has the singularity at $t=i \theta$. As shown in this paper, when the two singularities coincide

$$
\operatorname{Im} t_{s}(E)=\theta
$$

the effect of an external signal on tunneling enhances. Under the condition (3) the perturbation theory breaks down at essentially weaker non-stationary signal comparing to a general case. The parameter $\operatorname{Im} t_{s}(E)$ depends on properties of the static barrier and the particle energy [15], [16], but $\theta$ is a characteristic of a non-stationary signal. The physical meaning of the condition (3) is not straightforward since a quantum mechanical process is described 
by a product of amplitudes but not of probabilities. The condition (3) rather corresponds to a coherent cooperation of tunneling and quanta absorption, in other words, it is some "resonance" condition between motion of system and external signal. Eq. 3 determines some remarkable threshold energy $E_{T}$. As shown below, when the particle energy is big $E>E_{T}$ the tunneling process is moderately violated by the signal (2) (of course, if the signal is less, than the static field of the barrier $V / a$ ); for lower energy $E<E_{T}$ the process of the barrier transition is strongly stimulated even by a relatively small signal. This opens a possibility to manipulate effectively a tunneling process by a specially adapted (according to Eq. 3) electromagnetic signal of a small amplitude.

In Sections II-VII the tunneling probability as a function of time is calculated for the triangular barrier. In Section VIII the method of complex trajectories is described. In Sections IX-X this method is applied to the triangular barrier to compare with the results obtained by the direct solution of the Hamilton-Jacobi equation. In Sections XI-XIV the method of complex trajectories is applied to a barrier given by an analytical function $V(x)$, when there is no simple solution of the Hamilton-Jacobi equation.

\section{TRIANGULAR BARRIER}

In this Section we consider decay of the metastable state in the potential

$$
V(x)=V-\mathcal{E}_{0}|x|-\sqrt{\frac{2(V-E)}{m}} \delta(x)
$$

under action of a non-stationary electric field $\mathcal{E}(t)$. In the limit $\mathcal{E}_{0} \rightarrow 0$ the energy $E$ corresponds to the bound state in the $\delta$-function potential well. The symmetric wave function $(\psi(x, t)=\psi(-x, t))$ can be written down in the form

$$
\psi(x, t)=\exp (i S(x, t)+i \sigma(x, t))
$$

where the classical action $S$ obeys the Hamilton-Jacobi equation at $x>0$

$$
\frac{\partial S}{\partial t}+\frac{1}{2 m}\left(\frac{\partial S}{\partial x}\right)^{2}+V-\mathcal{E}_{0} x-x \mathcal{E}(t)=0
$$


with the boundary condition

$$
\left(\frac{\partial S(x, t)}{\partial x}\right)_{x=0}=i \sqrt{2 m(V-E)}
$$

At $x=0$ one can impose the condition $S(0, t)=-E t$. The equation for $\sigma$ has the form

$$
\frac{\partial \sigma}{\partial t}+\frac{1}{m} \frac{\partial S}{\partial x} \frac{\partial \sigma}{\partial x}+\frac{1}{2 m}\left(\frac{\partial \sigma}{\partial x}\right)^{2}-\frac{i}{2 m} \frac{\partial^{2} \sigma}{\partial x^{2}}=\frac{i}{2 m} \frac{\partial^{2} S}{\partial x^{2}}
$$

with the boundary condition

$$
\left(\frac{\partial \sigma}{\partial x}\right)_{x=0}=0
$$

Equations (6) and (8) are exact ones. The solution of the Hamilton-Jacobi equation (6) can be found by conventional methods [17

$$
\begin{aligned}
S(x, t)= & -\frac{1}{2 m} \int_{t_{0}}^{t} d t_{1}\left(p+\mathcal{E}_{0} t_{1}+\int_{0}^{t_{1}} d t_{2} \mathcal{E}\left(t_{2}\right)\right)^{2} \\
& +\left(p+\mathcal{E}_{0} t+\int_{0}^{t} d t_{1} \mathcal{E}\left(t_{1}\right)\right) x+(V-E) t_{0}-V t
\end{aligned}
$$

The functions $p(x, t)$ and $t_{0}(x, t)$ have to be defined from the conditions $\partial S / \partial p=0$ and $\partial S / \partial t_{0}=0$ what gives the following expressions

$$
p(x, t)=i \sqrt{2 m(V-E)}-\mathcal{E}_{0} t_{0}-\int_{0}^{t_{0}} d t_{2} \mathcal{E}\left(t_{2}\right)
$$

and

$$
m x=\int_{t_{0}}^{t} d t_{1}\left(p+\mathcal{E}_{0} t_{1}+\int_{0}^{t_{1}} d t_{2} \mathcal{E}\left(t_{2}\right)\right)
$$

Eq. 12 has to be inserted into Eqs. 11 and 13, what results in the final expression for the action

$$
\begin{aligned}
S(x, t) & =-\frac{1}{2 m} \int_{t_{0}}^{t} d t_{1}\left(i \sqrt{2 m(V-E)}+\left(t_{1}-t_{0}\right) \mathcal{E}_{0}+\int_{t_{0}}^{t_{1}} d t_{2} \mathcal{E}\left(t_{2}\right)\right)^{2} \\
& +x\left(i \sqrt{2 m(V-E)}+\left(t-t_{0}\right) \mathcal{E}_{0}+\int_{t_{0}}^{t} d t_{1} \mathcal{E}\left(t_{1}\right)\right)+(V-E) t_{0}-V t
\end{aligned}
$$

where the function $t_{0}(x, t)$ is given by the equation

$$
m x=i\left(t-t_{0}\right) \sqrt{2 m(V-E)}+\left(t-t_{0}\right)^{2} \frac{\mathcal{E}_{0}}{2}+\int_{t_{0}}^{t} d t_{1}\left(t-t_{1}\right) \mathcal{E}\left(t_{1}\right)
$$


By means of the relation (a partial derivative is taken under the fixed lower index)

$$
\left(\frac{\partial}{\partial t}\right)_{x}+\frac{1}{m}\left(\frac{\partial S}{\partial x}\right)_{t}\left(\frac{\partial}{\partial x}\right)_{t}=\left(\frac{\partial}{\partial t}\right)_{t_{0}}
$$

the equation (8) for $\sigma$ in new variables $t_{0}$ and $t$ has the form

$$
\left(\frac{\partial \sigma}{\partial t}\right)_{t_{0}}-\frac{1}{4(V-E) F^{2}}\left(\frac{\partial \sigma}{\partial t_{0}}\right)_{t}^{2}+\frac{i}{4(V-E) F}\left(\frac{\partial}{\partial t_{0}} \frac{1}{F} \frac{\partial \sigma}{\partial t_{0}}\right)_{t}=\frac{1+h\left(t_{0}\right)}{F \tau_{00}}
$$

Here new notations are introduced

$$
F\left(t_{0}, t\right)=1+i \frac{t_{0}-t}{\tau_{00}}\left(1+h\left(t_{0}\right)\right) ; \quad h(t)=\frac{\mathcal{E}(t)}{\mathcal{E}_{0}} ; \quad \tau_{00}=\frac{\sqrt{2 m(V-E)}}{\mathcal{E}_{0}}
$$

In terms of new variables the boundary condition (9) reads

$$
\left(\frac{\partial \sigma\left(t_{0}, t\right)}{\partial t_{0}}\right)_{t_{0}=t}=0
$$

In semiclassical approximation $\sigma$ should be small comparing to a big classical action $S$ and it can be expanded into a series

$$
\sigma=\sigma_{1}+\sigma_{2}+\ldots
$$

which is produced by Eq. 16, where the last two terms in the left-hand side have to be considered as a perturbation. Now one can write

$$
\sigma_{n}\left(t_{0}, t\right)=\int_{0}^{t-t_{0}} d \eta \Phi_{n}\left(\eta, t_{0}\right)+\int_{0}^{t_{0}} d t_{1} \Phi_{n}\left(0, t_{1}\right)
$$

where

$$
\Phi_{1}\left(t-t_{0}, t_{0}\right)=\frac{1+h\left(t_{0}\right)}{F\left(t_{0}, t\right) \tau_{00}}
$$

and

$$
\Phi_{2}\left(t-t_{0}, t_{0}\right)=\frac{1}{4(V-E) F^{2}}\left(\frac{\partial \sigma_{1}}{\partial t_{0}}\right)_{t}^{2}-\frac{i}{4(V-E) F}\left(\frac{\partial}{\partial t_{0}} \frac{1}{F} \frac{\partial \sigma_{1}}{\partial t_{0}}\right)_{t}
$$

From Eqs. 20 and 21 one can obtain an explicit expression

$$
i \sigma_{1}\left(t_{0}, t\right)=-\frac{1}{2} \ln F\left(t_{0}, t\right)+\frac{i}{2 \tau_{00}} \int_{0}^{t_{0}} d t_{1}\left(1+h\left(t_{1}\right)\right)
$$


The expression for $\sigma_{2}$ can be easily obtained from Eqs. 20 and 22 but it is too bulk and we do not write it here. The main parametric estimate at $n \geq 1$

$$
i \sigma_{n} \sim \frac{1}{\left((V-E) \tau_{00}\right)^{n-1}}
$$

characterizes Eq. 19 as a typical semiclassical series since $(V-E) \tau_{00} \gg 1$. The pulse width is supposed to be order of $\tau_{00}$. Eqs. 13 and 14 for the classical action and Eqs. 19 - 23 for $\sigma$ enable to consider a decay of the metastable state under action of the non-stationary field $\mathcal{E}(t)$

\section{CAUSALITY}

Suppose a pulse of the electric field has the form

$$
\tilde{\mathcal{E}}(t)=\mathcal{E}(t) \Theta\left(t^{\prime}-t\right)+\mathcal{E}^{\prime}(t) \Theta\left(t-t^{\prime}\right)
$$

The equation (25) can be considered in the complex time if to represent $\Theta$-functions in the form

$$
\Theta(t)=\left(\frac{1}{1+\exp (-\lambda t)}\right)_{\lambda \rightarrow+\infty}
$$

With the definition (26) the $\Theta$-function can be treated as $\Theta($ Ret). As follows from here,

the function $\tilde{\mathcal{E}}(t)$ at the complex $t$-plane depends on $\mathcal{E}(t)\left(\mathcal{E}^{\prime}(t)\right)$ only to the left (right) of the vertical line Re $t=t^{\prime}$. If to chose the contours of integration in Eq. 13 to the left of the vertical line with the real part $t$, than the action $S(x, t)$ does not have an information how the non-stationary pulse behaves at moments later than $t$. This choice of contours of integration corresponds to the causality principle.

\section{CLASSICAL ACTION}

In this Section we consider only the classical action $S$ in Eq. 5. Under the signal (2) the imaginary part of $S$ reaches its minimum value at some moment of time resulting in a 
maximum of the decay rate. For this reason we consider first the action $S(x, 0)$ at $t=0$. For a pulse symmetric in time $t_{0}(x, 0)$ is an imaginary value and we introduce $\tau_{0}(x)=-i t_{0}(x, 0)$. At small amplitude of the signal (2) essential values of $\tau_{0}$ are close to $\theta$ and the new variable

$$
z=1-\frac{\tau_{0}(x)}{\theta}
$$

is convenient $(z \ll 1)$. Below only integer values $n \geq 3$ in Eq. 2 and $\theta<\tau_{00}$ are considered. It follows from Eqs. 13 and 14

$$
i \frac{\partial S(x, 0)}{\partial x}=-\left(\tau_{00}-\theta\right)\left(1-\left(\frac{z_{1}}{z}\right)^{n-1}\right) \mathcal{E}_{0} ; \quad z_{1}=\left(\frac{\mathcal{E} \theta}{2^{n}(n-1)\left(\tau_{00}-\theta\right) \mathcal{E}_{0}}\right)^{1 /(n-1)}
$$

and

$$
i \frac{\partial^{2} S(x, 0)}{\partial x^{2}}=\frac{\theta /\left(\tau_{00}-\theta\right)+\left(z_{2} / z\right)^{n}}{1-\left(z_{2} / z\right)^{n}} \frac{m}{\theta} ; \quad z_{2}=\left(\frac{\mathcal{E} \theta}{2^{n}\left(\tau_{00}-\theta\right) \mathcal{E}_{0}}\right)^{1 / n}
$$

In Eqs. 28 and 29 the amplitude of the signal $\mathcal{E}$ is supposed to be small leading to small $z_{1}$ and $z_{2}$. As follows from Eq. 14, the function $\tau_{0}(x)$ is determined by the relation

$$
\frac{\partial m x}{\partial \tau_{0}}=\left(\tau_{00}-\theta\right) \mathcal{E}_{0}-\frac{\mathcal{E} \theta}{2^{n} z^{n}}
$$

By means of Eqs. 28 and 29 one can find the coordinate dependence of the action $S$, which is shown in Fig. 1. Two branches in Fig. 1 in the limit $\mathcal{E}=0$ go over into conventional WKB wave functions $\exp \left( \pm \int|p| d x\right)$. At the lower branch, where $\partial S / \partial x=0$,

$$
i S\left(x_{1}, 0\right)=(V-E) \theta\left(1-\frac{\theta^{2}}{3 \tau_{00}^{2}}\right) ; \quad x_{1}=\frac{\mathcal{E}_{0} \theta^{2}}{2 m}
$$

At the common point, where $\partial^{2} S / \partial x^{2} \rightarrow \infty$,

$$
i S\left(x_{2}, 0\right)=(V-E) \theta\left(1-\frac{\theta}{\tau_{00}}\right)^{2} ; \quad x_{2}=\frac{\mathcal{E}_{0} \theta}{2 m}\left(2 \tau_{00}-\theta\right)
$$

Near the common point $x_{2}$ the deviation of the action from the value (32) is proportional to $\left(x_{2}-x\right)$ and in the second order to $\pm\left(x_{2}-x\right)^{3 / 2}$, what develops the two branches. 


\section{NON-SEMICLASSICAL CORRECTIONS}

For validity of the semiclassical approximation the inequalities

$$
|S| \gg\left|\sigma_{1}\right| \gg\left|\sigma_{2}\right| \gg \ldots
$$

should hold. Like in a static case, one can expect a violation of the semiclassical theory near the point $x=x_{1}$, where $\partial S / \partial x=0$, and the point $x=x_{2}$, where $\partial^{2} S / \partial x^{2} \rightarrow \infty$. Let us compare $\sigma$ with the classical action $S$ near these "dangerous" points.

Under the condition $z_{1} \ll z \ll z_{2}^{n /(n+2)}$, as follows from Eqs. 21.24,

$$
i \sigma_{1}(x, 0)=-\frac{1}{2} \ln \left(\left(1-\frac{\theta}{\tau_{00}}\right)\left(1-\left(\frac{z_{2}}{z}\right)^{n}\right)\right)-\frac{\theta}{2 \tau_{00}}
$$

and

$$
i \sigma_{2}(x, 0)=\frac{1}{48(V-E) \theta} \frac{3 n(n+1)+n(2 n-3)\left(z_{2} / z\right)^{n}}{z_{2}^{2}\left(1-\theta / \tau_{00}\right)\left(1-\left(z_{2} / z\right)^{n}\right)^{3}}\left(\frac{z_{2}}{z}\right)^{n+2}
$$

An $x$-dependence in the right hand sides of Eqs. 34 and 35 comes through $z$ according to Eq. 27. At the point $x=x_{1}\left(z=z_{1}\right)$

$$
i \sigma_{1}\left(x_{1}, 0\right)=-\frac{1}{2} \ln \left(\frac{n-1}{z_{1}}\left(1-\frac{\theta}{\tau_{00}}\right)\right)-\frac{1}{2}-\frac{i \pi}{2}
$$

and

$$
i \sigma_{2}\left(x_{1}, 0\right)=-\frac{1}{48(V-E) \theta z_{1}\left(1-\theta / \tau_{00}\right)}\left(8(n-1)\left(1-\frac{\theta}{\tau_{00}}\right)^{2}+\frac{n(2 n-3)}{n-1}\right)
$$

A particle positioned in the well corresponds to $S(0,0)$ at the upper branch in Fig. $1(z \sim 1)$. A particle leaves the barrier under the condition $\partial S / \partial x=0$ at the point $x=x_{1}$ of the lower branch in Fig. $1\left(z=z_{1}\right)$. One should be sure the points $x=0$ and $x=x_{1}$ relate to the same semiclassical solution, on the other words, it should be possible to find a way from 0 to $x_{1}$ with no violation of the semiclassical conditions (33). Between real points $z \sim 1$ and $z=z_{1}$

there is only one "dangerous" point $z=z_{2}$, where, according to Eq. 35, $\sigma \rightarrow \infty$ and the condition (33) breaks down. Nevertheless, the semiclassical approximation remains valid if 
the condition (33) holds on some contour $\left|z-z_{2}\right| \sim z_{2}$ around the point $z_{2}$ in the complex $z$-plane. The point $z_{2}\left(x=x_{2}\right)$ is a branch point of the action, which has a contribution proportional to $\left(x_{2}-x\right)^{3 / 2}$, like a turning point in a static problem. The sequence of the Stokes and anti-Stokes lines [19] going from this point is qualitatively the same as in a static case. The condition (33) on the above circle can be written in the following approximate form if to put $z \sim z_{2}$ in Eq. $35\left(\theta<\tau_{00}\right)$

$$
\left(\frac{\theta}{\tau_{00}-\theta}\right)^{n / 2-1} \frac{a_{n}}{((V-E) \theta)^{n / 2}} \ll \frac{\mathcal{E}}{\mathcal{E}_{0}} ; \quad 1 \ll(V-E) \theta
$$

In the relation (38) the numerical coefficient $a_{n} \sim 1$ at $n \sim 1$, but at big $n$ the coefficient $a_{n}$ increases, what prescribes to choose a not big $n$ for validity of the semiclassical approxi-

mation. The condition $\left|\sigma_{2}\right| \ll\left|\sigma_{1}\right|$ at the point $x=x_{1}$ is less rigorous. The semiclassical conditions (38) require the pulse amplitude $\mathcal{E}$ to be not small. Remarkably, this amplitude can be still less than the static barrier field $\mathcal{E}_{0}$. At lower $\mathcal{E}$, than one satisfying the relations (38), one should expect the perturbation theory to be applicable.

\section{FINITE TIME}

Eq. 14 determines the function $t_{0}(x, t)$ and for the signal (2) one can write at $x=x_{1}$ and $|t|<\theta$

$$
t_{0}(x, t)=i \tau_{1}+\frac{z_{1} \theta^{2}}{2\left(\tau_{00}-\theta\right)}\left(i \frac{t^{2}}{\theta^{2}}+\frac{t^{3}}{\theta^{3}}\right)
$$

According to the causality principle, the contour of integration in Eq. 13 should be to the left of the time $t$ and hence the condition $\operatorname{Re} t_{0}<t$ results in the restriction $t>0$. At $t<0$ the semiclassical approach in its present form is not valid since the integration penetrates "in the future" and this case requires further investigation. The time dependence of the classical action can be found from Eq. 10

$$
\operatorname{Im} \frac{\partial S(x, t)}{\partial t}=-\frac{1}{2 m} \operatorname{Im}\left(i \sqrt{2 m(V-E)}+\left(t-t_{0}\right) \mathcal{E}_{0}+\int_{t_{0}}^{t} d t_{1} \mathcal{E}\left(t_{1}\right)\right)^{2}
$$


At $x=x_{1}$ and $t=0$ the right hand side of Eq. 40 is zero. One can easily obtain

$$
\operatorname{Im} S\left(x_{1}, t\right)=\operatorname{Im} S\left(x_{1}, 0\right)+(n-1)(V-E) \frac{t^{4}}{\theta \tau_{00}^{2}}
$$

\section{THE TRANSITION PROBABILITY}

Suppose $w(t)$ is the probability to find a particle in the $\delta$-function potential and initially

$w$ equals unity. The continuity equation reads $\partial w / \partial t=-(2 / m) \operatorname{Im}\left(\psi^{*} \partial \psi / \partial x\right)$, where the right hand side is taken at $x=x_{1}$. Using expression for the wave function

$$
\psi(x, t)=\psi(0, t) \exp (i S(x, t)-i S(0, t)+i \sigma(x, t)-i \sigma(0, t))
$$

where

$$
\psi(0, t) \simeq(2 m(V-E))^{1 / 4} \exp (-i E t)
$$

and by means of Eqs. 42 and 43 one can obtain

$$
\frac{\partial w(t)}{\partial t}=-\sqrt{\frac{8(V-E)}{m}}\left(\frac{\partial \operatorname{Re} S}{\partial x} \exp (-2 \operatorname{Im}(S+\sigma))\right)_{x=x_{1}}
$$

Eqs. 11 and 13 give $\partial \operatorname{Re} S / \partial x=\mathcal{E}_{0} t$ at $x=x_{1}$ and the main time dependence in the exponential of Eq. 44 follows from $S\left(x_{1}, t\right)$ (Eq. 41). Collecting the all terms, one can obtain finally $\left(\theta<\tau_{00}\right)$

$$
\begin{aligned}
\frac{\partial w(t)}{\partial t}=- & \frac{2(V-E) t}{\mathrm{e}(n-1)^{n /(n-1)}\left(\tau_{00}-\theta\right)}\left(\frac{\mathcal{E} \theta}{2\left(\tau_{00}-\theta\right) \mathcal{E}_{0}}\right)^{1 /(n-1)} \exp \left(-2(n-1) \frac{(V-E) t^{4}}{\theta \tau_{00}^{2}}\right) \\
& \quad \exp \left(-2(V-E) \theta\left(1-\frac{\theta^{2}}{3 \tau_{00}^{2}}\right)\right)
\end{aligned}
$$

As discussed in Section VI, Eq. 45 is valid only at $t>0$. The semiclassical conditions (33) are supposed to hold. According to Eq. 45, the typical time scale of the output flux $\Delta t \sim$ $\left(\theta \tau_{00}^{2} /(V-E)\right)^{1 / 4}$ determines the uncertainty of energy of outgoing particles $\Delta E \sim \Delta t^{-1}$ which is much smaller than the energy $E$. The decay rate $\partial w(t) / \partial t$ tends to its static value at $t \rightarrow \pm \infty$ having a maximum at some moment of time

$$
\left(\frac{\partial w}{\partial t}\right)_{\max } \sim \exp \left(-2(V-E) \theta\left(1-\frac{\theta^{2}}{2 \tau_{00}^{2}}\right)\right)
$$


The exponent in Eq. 46, according to its derivation, is a minimum value of the imaginary part of the classical action. A minimum value of action can be calculated, as known from classical mechanics, by means of trajectories satisfying Newton's equation. So, when we are not interested in the full time dependence of a decay rate but we need only its maximum value with an exponential accuracy, the method of classical trajectories can be used. This method is described in the next Section.

\section{METHOD OF COMPLEX TRAJECTORIES}

In this Section we consider penetration of incident particles through a potential barrier under action of a non-stationary pulse. We restrict ourselves only by the main exponential approximation when one can use the semiclassical expression for a wave function $\psi(x, t) \sim$ $\exp (i S(x, t))$. We consider here a particle flux on to the barrier shown in Fig. 2, but the final result can be easily applied to decay of the metastable state through the triangular barrier (田). The maximum value of the outgoing flux of particles can be calculated as a maximum with respect to time

$$
W_{\max } \sim \max \left|\exp \left(i S(x, t)-i S\left(x_{0}, t\right)\right)\right|^{2}
$$

Here $x$ is some coordinate to the right of the barrier, $x_{0} \rightarrow-\infty$, where $\operatorname{Im} S\left(x_{0}, t\right)=0$, and in Eq. 47 one can put $S\left(x_{0}, t_{0}\right)$ instead of $S\left(x_{0}, t\right)$. The right hand side of Eq. 47 does not depend on $x$ and $x_{0}$. It is a function of $t$ only. Eq. 47 corresponds to the extreme classical action, which can be found by method of classical trajectories $x(t)$ defined in the complex $t$-plane, since in real time there is no classical trajectory for an under-barrier motion. The

complex path $C$ is shown in Fig. 3. The real classical turning point is $x_{1}=x\left(t_{1}\right)$, where $\partial x(t) / \partial t=0$. This point corresponds to the classical exit of a particle from under the barrier. The real coordinate $x_{0}=x\left(\tilde{t}_{0}\right)$ is defined under the condition $\operatorname{Re} \tilde{t}_{0}=t_{0} \rightarrow-\infty$. The classical trajectory connects the points $\left\{x_{0}, \tilde{t}_{0}\right\}$ and $\left\{x_{1}, t_{1}\right\}$. The contour $C$ is symmetric 
with respect to the real axis. It is convenient to write Eq. 47 in the form

$$
W_{\max } \sim\left|\exp \left(i S\left(x_{1}, t_{1}\right)-i S\left(x_{0}, t_{0}\right)\right)\right|^{2}
$$

Since at $t \rightarrow-\infty$ the non-stationary field $\mathcal{E}(t) \rightarrow 0$, a connection between values of the action at the points $t_{0}$ and $\tilde{t}_{0}$ is simple

$$
S\left(x_{0}, t_{0}\right)=S\left(x_{0}, \tilde{t}_{0}\right)+\left(\tilde{t}_{0}-t_{0}\right) E
$$

where $E$ is the energy of an incident particle. According to Eqs. 48 and 49, the maximum amplitude value of the outgoing flux of particles has now the form

$$
W_{\text {max }} \sim \exp (-A)
$$

where

$$
A=-i \int_{C} d t\left(\frac{m}{2}\left(\frac{\partial x}{\partial t}\right)^{2}-V(x)+x \mathcal{E}(t)+E\right)
$$

is defined by means of trajectory satisfying Newton's equation $m \partial^{2} x / \partial t^{2}+V^{\prime}(x)=\mathcal{E}(t)$ in the complex time. Due to symmetry of the contour $C$ the value of $A$ is real. The trajectory $x(t)$ should not be necessary real at all $t$, it should be real at least in vicinities of real points $x_{0}$ and $x_{1}$. On the left horizontal parts of the contour $C$, where $\mathcal{E}(t)=0, x(t)$ satisfies the equation

$$
\frac{m}{2}\left(\frac{\partial x}{\partial t}\right)^{2}+V(x)=E
$$

and is expressed through the real functions $x=f\left(t-\tilde{t}_{0}, E\right)(\mathrm{up})$ and $x=f\left(t-\tilde{t}_{0}^{*}, E\right)($ down), where $E$ is a real energy. Now one can formulate conditions how to choose the contour $C$ : for given (at $t \rightarrow-\infty$ ) particle energy $E$ and the pulse shape $\mathcal{E}(t)$ one should find $\operatorname{Im} \tilde{t}_{0}$ and the real turning point $x_{1}=x\left(t_{1}\right)$. Eq. 51 holds for a potential barrier $V(x)$, which is an analytical function of the variable $x$. Such a barrier has no artificial restriction in coordinate (no singularity at a real $x$ ). For this reason, the equation (51) can be interpreted as one accounting not only an under-barrier part but also some pre-barrier motion. 


\section{APPLICATION TO A TRIANGULAR BARRIER}

Eq. 51 is applicable to the case of a potential barrier $V(x)$, which is an analytical function of the variable $x$. In the case of the triangular barrier (四), which is a non-analytical function, the all classical path of the particle is restricted by an under-barrier motion. In this case the exponent $A$, instead of Eq. 51, should be written in the form

$$
A=2 \operatorname{Im} \int_{i \tau_{0}}^{0} d t\left(\frac{m}{2}\left(\frac{\partial x}{\partial t}\right)^{2}-V+x \mathcal{E}_{0}+x \mathcal{E}(t)+E\right)
$$

where $x(t)$ is the trajectory satisfying the Newton equation in the complex time

$$
m \frac{\partial^{2} x}{\partial t^{2}}-\mathcal{E}_{0}=\mathcal{E}(t)
$$

The trajectory starts at the metastable well $x\left(i \tau_{0}\right)=0$ with the boundary conditions

$$
\left(\frac{\partial x(t)}{\partial t}\right)_{i \tau_{0}}=i \sqrt{\frac{2(V-E)}{m}}
$$

For a symmetric pulse $\mathcal{E}(-t)=\mathcal{E}(t)$ the velocity $\partial x / \partial t=0$ at $t=0$, at this point the

particle escape from under the barrier, and this terminates the integration in Eq. 53. The parameter $\tau_{0}$ has a meaning of under-barrier traversal time [18] and can be found from the equation

$$
\mathcal{E}_{0} \tau_{0}+\int_{0}^{\tau_{0}} d \tau \mathcal{E}(i \tau)=\sqrt{2 m(V-E)}
$$

Eq. 56 is equivalent to the condition $p=0$ following from Eq. 11. Since the time is imaginary the function $A$ can be called the Eucledian action

$$
A=2(V-E) \tau_{0}-\frac{\mathcal{E}_{0}^{2}}{3 m} \tau_{0}^{3}-\frac{2 \mathcal{E}_{0}}{m} \int_{0}^{\tau_{0}} \tau d \tau \int_{0}^{\tau} d \tau_{1} \mathcal{E}\left(i \tau_{1}\right)-\frac{1}{m} \int_{0}^{\tau_{0}} d \tau\left(\int_{0}^{\tau} d \tau_{1} \mathcal{E}\left(i \tau_{1}\right)\right)^{2}
$$

The outgoing particle has the energy $E+\delta E$, where

$$
\delta E=V-E-\left(\mathcal{E}_{0}+\mathcal{E}(0)\right)\left(\frac{\mathcal{E}_{0}}{2 m} \tau_{0}^{2}+\frac{1}{m} \int_{0}^{\tau_{0}} d \tau \int_{0}^{\tau} d \tau_{1} \mathcal{E}\left(i \tau_{1}\right)\right)
$$

After escape the barrier an action of the non-stationary field on the particle can be omitted since it is determined by the parameter $\mathcal{E} / \mathcal{E}_{0}$ which is much smaller than one governing 
the particle under the barrier and defined by the conditions (38). In the absence of a nonstationary pulse the energy of outgoing particles has the same value $(\delta E=0)$ and the Eucledian action equals $A_{0}$ determined by the conventional WKB formula

$$
A_{0}(E)=\frac{4}{3}(V-E) \tau_{00}
$$

where $\tau_{00}$ is given by Eq. 17 and has a meaning of the under-barrier traversal time in the stationary case. The condition $\theta=\tau_{00}$, which is a particular case of Eq. 3, sets some threshold energy

$$
E_{T}=V-\frac{\theta^{2} \mathcal{E}_{0}^{2}}{2 m}
$$

As follows from Eqs. 57 and 58, the intensity and the energy of outgoing particles strongly depends on whether the initial energy $E$ bigger $\left(\tau_{00}<\theta\right)$ or smaller $\left(\theta<\tau_{00}\right)$ than $E_{T}$. At

$E_{T}<E$ the effect of the non-stationary signal on tunneling is weak and increases only in the vicinity of $E_{T}$

$$
A=A_{0}\left(1-\frac{3 \mathcal{E}}{(n-1) 2^{n} \mathcal{E}_{0}} \frac{1}{\left(1-\tau_{00} / \theta\right)^{n-2}}\right) ; \quad \frac{\delta E}{V-E}=\frac{2 \mathcal{E}}{(n-1) 2^{n} \mathcal{E}_{0}} \frac{1}{\left(1-\tau_{00} / \theta\right)^{n-1}}
$$

At low energies $E<E_{T}$ the situation is very non-perturbative

$$
A=A_{0}\left(E_{T}\right)+2\left(E_{T}-E\right) \theta ; \quad \delta E=E_{T}-E
$$

what coincides with the exponent in Eq. 46 obtained by a direct solution of the HamiltonJacobi equation. The energy dependence of the Eucledian action is shown in Fig. 4. This type of scenario of barrier penetration is shown schematically in Fig. 2 in the case of particle flux on the barrier.

\section{SEPARATION OF QUANTA ABSORPTION AND TUNNELING}

For a monochromatic field $\mathcal{E}_{\omega}$ of frequency $\omega$ the total probability of penetration through a barrier can be approximately written as a product of two probabilities: absorption of $N$ 
quanta and tunneling (see the comment to Eq. 1)

$$
\frac{\partial w}{\partial t} \sim\left(\frac{\mathcal{E}_{\omega}}{\mathcal{E}_{0} \omega \tau_{00}}\right)^{2 N} \exp \left(-A_{0}(E+\omega N)\right)=\exp (-A(\omega, N))
$$

For the pulse (1) the amplitude $\mathcal{E}_{\omega}$ should be substituted by $\mathcal{E}(\omega \theta)^{n-1} \exp (-\omega \theta)$ and the effective action becomes of the form

$$
A(\omega, N)=A_{0}(E+\omega N)+2 \ln \left(\frac{\mathcal{E}_{0}}{\mathcal{E}(\omega \theta)^{n-2} \exp (-\omega \theta)}\right)
$$

Semiclassical approximation corresponds to some optimum choice of $\omega$ in a continuous spectrum of the pulse and the number of quanta of this optimum frequency $N$, which provide a minimum of $A(\omega, N)$. The condition $\partial A(\omega, N) / \partial N=0$ gives the following relations

$$
\begin{aligned}
& \delta E=\omega N=\left(E_{T}-E\right)-\frac{2 \theta(V-E)}{\omega \tau_{00}} \ln \left(\frac{\mathcal{E}_{0}}{\mathcal{E}(\omega \theta)^{n-2}}\right) \\
& A=A_{0}\left(E_{T}\right)+2\left(E_{T}-E\right) \theta-\frac{2(V-E)}{\omega}\left(1-\frac{\theta^{2}}{\tau_{00}^{2}}\right) \ln \left(\frac{\mathcal{E}_{0}}{\mathcal{E}(\omega \theta)^{n-2}}\right)
\end{aligned}
$$

The further minimization, with respect to $\omega$, give an infinite (in this approach) value of $\omega$ indicating the logarithmic terms in Eqs. 65 to be small and hence Eqs. 65 coincide with the result $(62)$.

Let us consider another example, when such simple approach also give a correct (with an exponential accuracy) decay rate. Suppose the Gaussian pulse

$$
\mathcal{E}(t)=\mathcal{E} \exp \left(-\Omega^{2} t^{2}\right)
$$

acts on a particle in the stable potential well (丑) with $\mathcal{E}_{0}=0$. Than in the effective action

$$
A(\omega, N)=2 N \ln \frac{\omega \sqrt{m(V-E)}}{\mathcal{E}_{\omega}}
$$

one should put $\mathcal{E}_{\omega} \rightarrow \mathcal{E} \exp \left(-\omega^{2} / \Omega^{2}\right)$, according to the Fourier harmonic of the pulse, and $N=(V-E) / \omega$, since in this case there is no tunneling and a particle should reach the top of the barrier. This leads to the relation

$$
A\left(\omega, \frac{V-E}{\omega}\right)=2(V-E)\left(\frac{\omega}{4 \Omega^{2}}+\frac{1}{\omega} \ln \frac{\omega \sqrt{m(V-E)}}{\mathcal{E}}\right)
$$


The minimization of this expression with respect to $\omega$ gives the optimum value of $A$

$$
A=\frac{2(V-E)}{\Omega}\left(\ln \frac{\Omega \sqrt{m(V-E)}}{\mathcal{E}}\right)^{1 / 2}
$$

the optimum pulse frequency, and the optimum number of absorbed quanta

$$
\omega_{\text {opt }}=2 \Omega\left(\ln \frac{\Omega \sqrt{m(V-E)}}{\mathcal{E}}\right)^{1 / 2} ; \quad N_{\text {opt }}=\frac{V-E}{\omega_{\text {opt }}}
$$

The result (69) coincides with calculation of the Eucledian action for the pulse (66) by semiclassical methods developed above (calculations are not put in this paper). One can see from here, the decay rate under action of a non-stationary pulse can be calculated with an exponential accuracy on the base of simple arguments of optimum frequency and number of quanta. This approach of separation of quanta absorption and subsequent tunneling, described in this Section, works only for a potential $V(x)$ which is not an analytical function of the variable $x$ like the potential (画. In this case one can use an interpretation of quanta

absorption at some point $x$ (position of singularity of $V(x)$ on the real axis). When $V(x)$ is an analytical function there is no such particular point, the situation is more complicated, and the method of simple separation of absorption and tunneling does not work, since the quantum interference of these processes becomes very non-trivial. The case of analytical potential is considered in the next Section.

\section{WEAK NON-STATIONARY SIGNAL}

Let us go back to an analytical potential barrier $V(x)$. When $\mathcal{E}(t)=0$ the contour $C$ is reduced to the contour $C_{0}$ shown in Fig. 3, which consists of the vertical part between the points $\pm i \operatorname{Im} \tilde{t}_{0}$ and the horizontal semi-infinite lines at Ret $<0$. In this static case

$$
\operatorname{Im} \tilde{t}_{0}=\sqrt{\frac{m}{2}} \int \frac{d x}{\sqrt{V(x)-E}}
$$

where the integration goes between two classical turning points determined by the relation $V(x)=E$. Eq. 51 at $\mathcal{E}(t)=0$ determines the conventional WKB exponent by means of the 
unperturbed Lagrangian $L_{0}$

$$
\begin{aligned}
& A_{0}=-i \int_{C} d t L_{0}=2 \sqrt{2 m} \int d x \sqrt{V(x)-E} \\
& L_{0}=\frac{m}{2}\left(\frac{\partial x_{0}}{\partial t}\right)^{2}-V\left(x_{0}\right)+E
\end{aligned}
$$

Here $x_{0}(t)$ is the classical trajectory determined at all $t$ by Eq. 52. The small pulse $\mathcal{E}(t)$ results in the perturbed trajectory $x_{0}(t)+\delta x(t)$. The perturbation in Eq. 51 has the form

$$
A=-i \int_{C} L_{0}+\int_{C} x_{0}(t) \mathcal{E}(t)+m\left(\frac{\partial x_{0}}{\partial t} \delta x\right)\left(\tilde{t}_{0}^{*}\right)-m\left(\frac{\partial x_{0}}{\partial t} \delta x\right)\left(\tilde{t}_{0}\right)
$$

The velocities $\partial x / \partial t$ at $\tilde{t}_{0}^{*}$ and $\tilde{t}_{0}$ are real and

$$
\operatorname{Im} \delta x\left(\tilde{t}_{0}\right)=-\left(\frac{\partial x_{0}}{\partial t} \delta \tilde{t}_{0}\right)\left(\tilde{t}_{0}\right)
$$

Here $\delta \tilde{t}_{0}$ is a variation of $\tilde{t}_{0}$ due to the pulse given by Eq. 71. One can easily see that

$$
\int_{C} d t L_{0}=\int_{C_{0}} d t L_{0}-2 i\left(\frac{\partial x_{0}}{\partial t}\right)^{2}\left(\tilde{t}_{0}\right) \operatorname{Im} \delta \tilde{t}_{0}
$$

Collecting Eqs. 73775, one can obtain

$$
A=A_{0}+\delta A ; \quad \delta A=-i \int_{C} d t \mathcal{E}(t) x_{0}(t+\Delta t)
$$

Here we keep the argument shift $\Delta t$ of the unperturbed solution, satisfying Eq. 52, determined in the way the classical turning point $t_{1}=-\Delta t$. The method of classical trajectories produces a minimum value of $A$, this means the shift $\Delta t$ to be found from the minimization condition

$$
\frac{\partial \delta A}{\partial \Delta t}=0
$$

A meaning of the minimization condition (77) can be clarified in the following way. According to classical mechanics, the variation of the particle energy is $\partial E / \partial t=\mathcal{E}(t) \partial x_{0} / \partial t$. At $t=\tilde{t}_{0}$ and at $t=\tilde{t}_{0}^{*}$, when the non-stationary field is zero, energy should have the same values, that is

$$
\int_{C} d t \mathcal{E}(t) \frac{\partial x_{0}(t+\Delta t)}{\partial t}=0
$$


Eq. 78 coincides with Eq. 77. If the minimization condition (77) violates the particle energy $E$ would acquire an imaginary part. In summary of this Section, in case of small non-stationary field $\mathcal{E}(t)$ one can use the perturbation approach (76) with the further minimization (78).

\section{ANALYTICAL PROPERTIES OF TRAJECTORIES}

We consider the potential barrier

$$
V(x)=\frac{V}{\cosh ^{2} x / a}
$$

The classical unperturbed trajectory satisfies the relations [15,16]

$$
\frac{\partial x_{0}(t+\Delta t)}{\partial t}=\frac{a \omega \sinh \omega(t+\Delta t)}{\sqrt{\cosh ^{2} \omega(t+\Delta t)+E /(V-E)}} ; \quad \omega^{2}=\frac{2 E}{m a^{2}}
$$

and is an analytical function of the complex variable $t$ having the branch points at $t=t_{s}, t_{s}^{*}$ where

$$
t_{s}=i \tau_{s}-\frac{1}{\omega} \ln \frac{\sqrt{V}+\sqrt{E}}{\sqrt{V-E}}-\Delta t ; \quad \tau_{s}=\frac{\pi}{2 \omega}
$$

Close to the branch point $t_{s}$ the trajectory has the form

$$
x_{0}(t+\Delta t)=-\frac{i \pi a}{2}+a \sqrt{2 \omega\left(t_{s}-t\right) \sqrt{V / E}}
$$

The cut is shown in Fig. 3 by the dashed horizontal line. Now the integral, defining $\delta A$ in Eq. 76, can be calculated on base of analytical properties.

\section{TUNNELING PROBABILITY}

Let us choose the non-stationary pulse in the form (2) with $n=2$ and the potential barrier (79). Then the integrand in Eq. 76 has singularities of two types in the complex $t$-plane:

$t_{s}$ comes from the analytical function $x_{0}(t+\Delta t)$ and $i \theta$ comes from the analytical function $\mathcal{E}(t)$. There are different positions of the contour $C$ with respect to those singular points 
giving rise to different branches of the extreme action. The branch, giving the minimum value of $A$, results from the position shown in Fig. 3, when the contour $C$ goes between the two singularities. This very branch determines the effect. We consider here only the case $\operatorname{Im} t_{s}<\theta$. The contour $C$ can be deformed up and in the limit $\left(\theta-\tau_{s}\right) \ll \theta$ the pole circle at $t=i \theta$ gives the main contribution

$$
\delta A=\pi \mathcal{E} a \tau_{s}^{2}\left(\frac{V}{E}\right)^{1 / 4} \operatorname{Re} \sqrt{\frac{\omega}{2\left(t_{s}-i \theta\right)}}
$$

According to Eq. 81, $t_{s}$ depends on $\Delta t$ and the minimization (77) produces

$$
\Delta t=-\frac{\theta-\tau_{s}}{\sqrt{3}}
$$

The moment $t_{1}=-\Delta t$ is the delay time of outgoing particles from under the barrier. The correction $\delta A$ in Eq. 76 has the form

$$
\delta A=-\frac{\pi}{4} \mathcal{E} a \tau_{s}^{2}\left(\frac{3 V}{E}\right)^{1 / 4} \sqrt{\frac{3 \omega}{\theta-\tau_{s}}}
$$

Finally, Eqs. 50, 72, 76, and 85 determine the maximum outgoing flux of particles tunneling through the potential barrier (79) in the case of a weak non-stationary signal (2) with $n=2$. Eq. 85 is analogous to the formula (61) for the triangular potential. When $\theta \rightarrow \operatorname{Im} t_{s}$ the perturbation theory breaks down and the result becomes to be very non-linear function of the non-stationary pulse like Eq. 62. We see that the relation (3) plays a crucial role

in physics of tunneling under non-stationary conditions. For the potential barrier $(\overline{79}) t_{s}$ appears in a "natural" way as a result of analytical properties, whereas for a non-analytical potential it is determined by the time of motion between a turning point and a point of the non-analytical singularity of the potential $\left(\tau_{00}\right.$ in the case of the triangular potential (田)).

\section{DISCUSSIONS AND CONCLUSIONS}

In the case of the triangular potential barrier the semiclassical theory in a non-stationary case is constructed based on classical trajectories in the complex time. This becomes possible since for such barrier non-semiclassical corrections can be calculated exactly. The conditions 
of applicability of the semiclassical approach (softly varying and not very small pulse) are not surprising. Much less trivial matter is the way of construction of the trajectory method. The condition (3) of coincidence of singularity of a trajectory and of a field leads to unexpected conclusions. The threshold energy $E_{T}$, set by the condition (3), divides the all incident flux of particles by two groups: (i) particles with $E>E_{T}$ passes the barrier practically like in a stationary case, (ii) particles with lower energies $E<E_{T}$ are affected strongly by the pulse which collects all particles after passing the barrier at the same energy $E_{T}$ regardless their initial energy. This enables to use short pulses, adapted to the necessary energy level by the relation (3), to get outgoing particles collected at this level at some moment of time. In other words, adapted signals can be used for a selective control of tunneling: in solids and molecules it excites only some particular bonds leaving other bonds non-excited. There is an obvious advantage of use soft signals for control of tunneling. The hard pulse of the type (21) cannot control tunneling selectively, since it kicks up all particles to the top of the barrier. When the pulse (2) serves as an envelope for the monochromatic signal of the frequency $\Omega \sim 1 / V$ it provides in a non-selective manner equal $\Omega$-shifts of all escaping particles with respect to their incident energies. It is remarkable, the soft Gaussian signal $\mathcal{E}(t)=\mathcal{E} \exp \left(-t^{2} / \theta^{2}\right)$ cannot produce a strong enhancement of tunneling when its amplitude is much less than a static field of a potential barrier. This is an indication of importance of the analytical structure of the signal (2). This can be understood in another way looking at Eq. 1, where the Fourier harmonics of the Gaussian signal $\mathcal{E}_{\Omega} \sim \mathcal{E} \exp \left(-\Omega^{2} \theta^{2} / 4\right)$ do not result in divergence in $\Omega$.

The static electric field in solids and molecules can be estimated as $\mathcal{E}_{0} \sim 10^{7} \mathrm{~V} / \mathrm{cm}$, a typical pulse width is in the range of tens of femtoseconds, and the amplitude of the electric field of the pulse can be chosen as $\mathcal{E} \sim 10^{4}-10^{5} \mathrm{~V} / \mathrm{cm}$, what is reachable in experiments.

The developed theory is also applicable to quantum mechanical over-barrier reflection of particles. The reflection of classical waves (electromagnetic, hydrodynamic, etc.) from a spatially-smooth medium also may be described by the above theory, when the medium is influenced by an adapted signal. 


\section{ACKNOWLEDGMENT}

I am grateful to S. Obukhov, J. Krause, A. Efimov, and E. Ryabov for stimulating discussions 


\section{REFERENCES}

[1] W. S. Warren, H. Rabitz, and M. Dahlen, Science 259, 1581 (1993)

[2] S. Shi and H. Rabitz, J.Chem.Phys. 92, 364 (1990)

[3] R. S. Judson and H. Rabitz, Phys.Rev.Lett. 68, 1500 (1992)

[4] B. Kohler, J. L. Krause, F. Raksi, K. R. Wilson, V. V. Yakovlev, R. M. Whitnel, and Y. Yan, Accounts of Chemical Research, 28, 133 (1995)

[5] D. W. Schumacher, J. H. Hoogenraad, D. Pinkos, and P. H. Bucksbaum, Phys.Rev. A $52,4719(1995)$

[6] J. L. Krause, D. H. Reitze, G. D. Sanders, A. V. Kusnetsov, and C. J. Stanton, Phys.Rev. B 57, 9024 (1998)

[7] R. Atanasov, A. Hache, J. L. P. Hufges, H. M. van Driel, and J. E. Sipe, Phys.Rev.Lett. 76, $1703(1996)$

[8] A. M Weiner, J. P. Heritage, and Kirschner, J.Opt.Soc.Am. B 5, 1563 (1988)

[9] A. E. Efimov, C. Schaffer, and D. H. Reitze, J.Opt.Soc.Am. B 12, 1968 (1995)

[10] Quantum Tunneling in Condensed Media, edd. by A. Leggett and Yu. Kagan, North Holland, Amsterdam, 1992

[11] P. Hanggi, P. Talkner, and M. Borkovec, Rev.Mod.Phys. 62, 251 (1990)

[12] S. Keshavamurthy and W. H. Miller, Chem.Phys.Lett. 218, 189 (1994)

[13] N. T. Maitra and E. J. Heller, Phys.Rev.Lett. 78, 3035 (1997)

[14] J. Anker and H. Grabert, Europhys.Lett. 47, 285 (1999)

[15] B. I. Ivlev and V. I. Melnikov, Phys.Rev.Lett. 55, 1614 (1985)

[16] B. I. Ivlev and V. I. Melnikov, Zh.Eksp.Teor.Fiz. 90, 2208 (1986) [Sov.Phys.JETP 63, 
$1295(1986)]$

[17] L. D. Landau and E. M. Lifshitz, Quantum Mechanics, Pergamon Press, 1977

[18] M. Buettiker and R. Landauer, Phys.Rev.Lett 49, 1734 (1982)

[19] J. Heading, An Introduction to Phase-Integral Methods, Methuen, John Wiley, 1962 


\section{FIGURES}

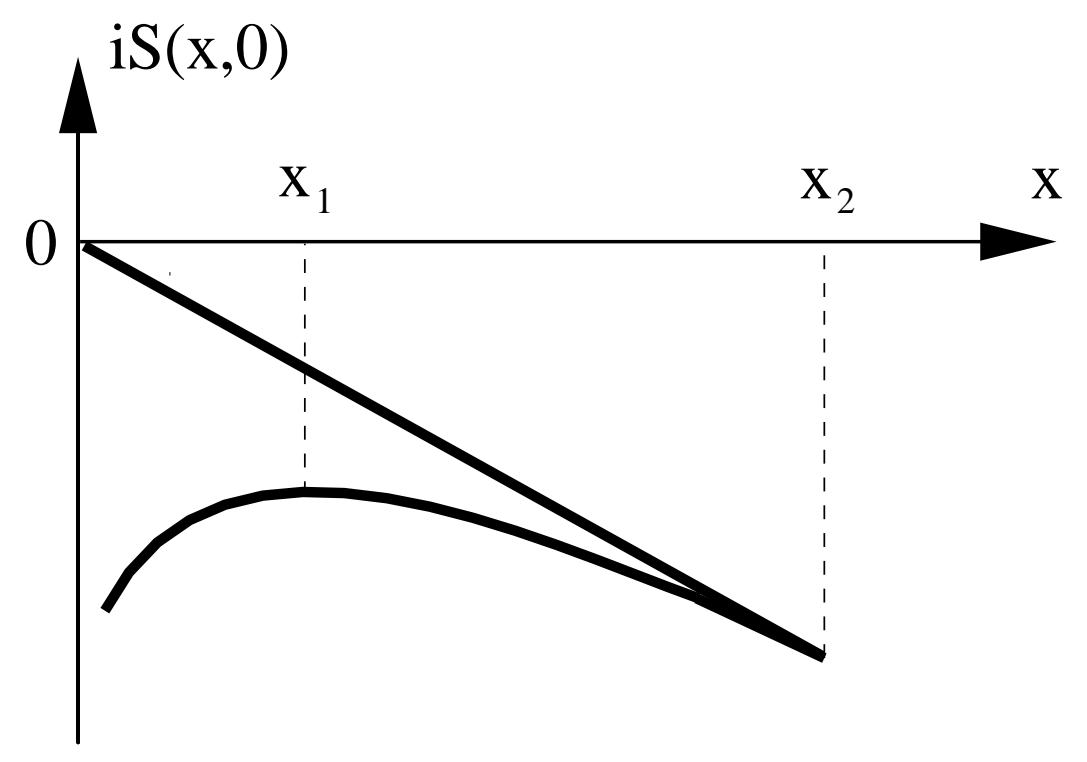

FIG. 1. A plot of the imaginary part of the action for the case of the triangular potential barrier at $t=0$. The classical position of a particle before tunneling is $x=0$ and after tunneling is $x=x_{1}$ (a classical turning point). In absence of a non-stationary pulse the two branches go over into conventional increasing and decreasing WKB branches. 


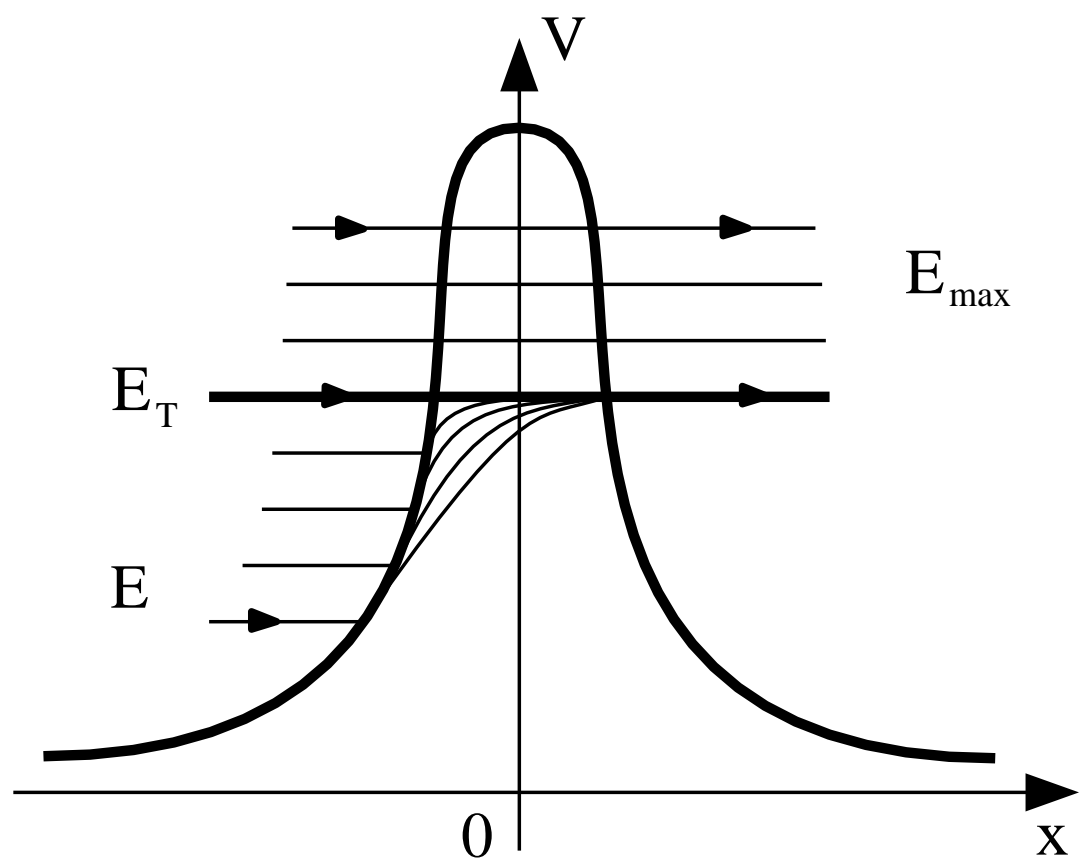

FIG. 2. A snap-shot of a particle motion through the potential barrier under the action of a non-stationary pulse at a moment of the maximum tunneling flux. $E_{\max }$ is the maximum (in time) energy of escaped particles. All particles with the incident energies $E<E_{T}$ are collected at the threshold level $E_{T}$ after passing the barrier. A motion of particles with $E>E_{T}$ is violated a little. 


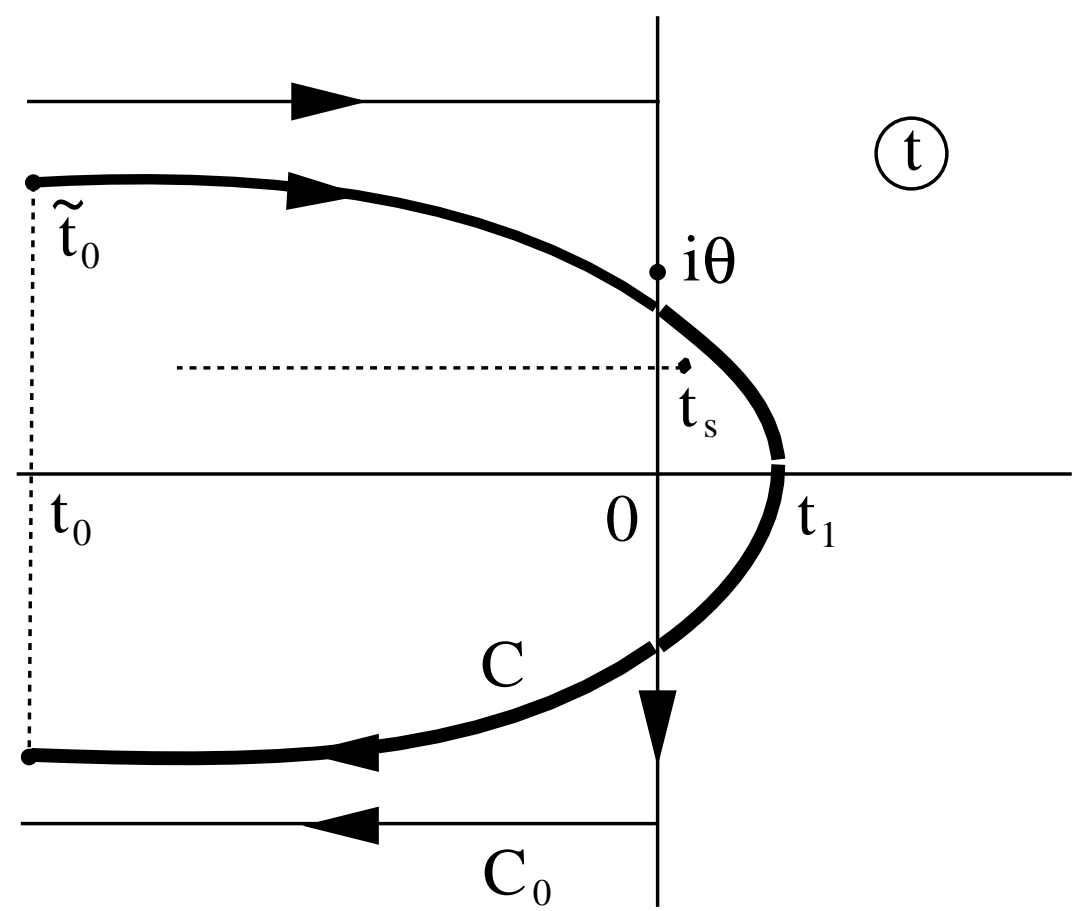

FIG. 3. The contours of integration $C$ and $C_{0}$ (in absence of a pulse) are shown in the plane of complex time. $i \theta$ is the position of singularity of the non-stationary pulse and $t_{s}$ is the branch point singularity of the classical trajectory. The cut is denoted by the dashed horizontal line. 


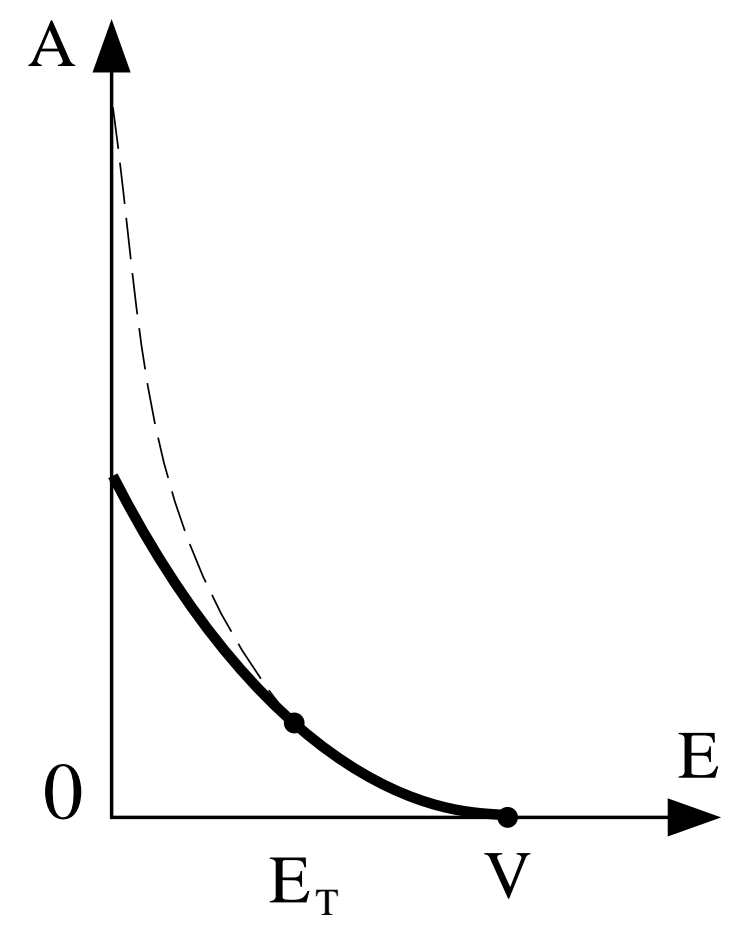

FIG. 4. The energy dependence of the exponent $A(E)$ (solid line) which determines the maximum (in time) of the tunneling probability $W_{\max } \sim \exp (-A(E))$. The dashed line is the plot of the exponent $A_{0}(E)$ in the absence of a pulse. It merges the solid line at $E>E_{T}$. 